\title{
The Spectral Theory of the Vibrating Periodic Beam
}

\author{
Vassilis G. Papanicolaou \\ Department of Mathematics and Statistics, Wichita State University, Wichita, KS 67260-0033, \\ USA
}

Received: 25 May 1994/in revised form: 8 September 1994

Abstract: We study the spectral theory of the fourth-order eigenvalue problem

$$
\left[a(x) u^{\prime \prime}(x)\right]^{\prime \prime}=\lambda \rho(x) u(x), \quad-\infty<x<\infty,
$$

where the functions $a$ and $\rho$ are periodic and strictly positive. This equation models the transverse vibrations of a thin straight (periodic) beam whose physical characteristics are described by $a$ and $\rho$.

We examine the structure of the spectrum establishing the fact that the periodic and antiperiodic eigenvalues are the endpoints of the spectral bands. We also introduce an entire function, which we denote by $E(\lambda)$, connected to the spectral theory, whose zeros (at least the ones of odd multiplicity) are shown to lie on the negative real axis, where they define a collection of "pseudogaps." Next we prove some inverse results in the spirit of two old theorems of Borg for the Hill's equation. We finish with a "determinant formula" (i.e. a multiplicative trace formula) and some comments on its role in the formulation of the general inverse problem.

\section{Introduction}

The Euler-Bernoulli equation for the free undamped infinitesimal transverse vibrations of a thin, straight beam can be written as (see [T-Y] or [G])

$$
\left[a(x) u^{\prime \prime}(x)\right]^{\prime \prime}=\lambda \rho(x) u(x),
$$

where $u$ is the deflection of the beam and the positive functions $a$ and $\rho$ correspond to physical characteristics of the beam.

Mathematically speaking, the Euler-Bernoulli equation (together with appropriate boundary conditions) is a fourth-order eigenvalue problem. In contrast with the second-order case, works on fourth-order problems appear sparsely in the literature. Joyce McLaughlin (see [Mc] or [G]; also [B] for some relevant results) has solved an inverse problem, where the beam equation is considered on a finite interval with certain separated boundary conditions. A variant of this problem was recently solved by V.A. Yurko (see [Y1]). 
This work is concentrated on the periodic case, namely

$$
\left(a u^{\prime \prime}\right)^{\prime \prime}=\lambda \rho u, \quad a(x+b)=a(x), \quad \rho(x+b)=\rho(x), \quad-\infty<x<\infty .
$$

Notice that $a$ and $\rho$ are assumed twice continuously differentiable and strictly positive. Without loss of generality we impose the following normalizations:

$$
\int_{0}^{b}\left[\frac{\rho(x)}{a(x)}\right]^{1 / 4} d x=b, \quad a(0)=1 .
$$

Elastic structures consisting of many thin elements arranged periodically are common in applications. Although there are some authors that have studied such problems numerically (see for example [M]), as far as we know, nobody has studied (1) theoretically.

Our goal here is to understand the spectral theory of (1) and to start developing a feeling of how to formulate the inverse problem. For its second-order analog, namely the Hill's equation, the corresponding literature is huge. Also, there are many known results for the general linear ordinary differential equation with periodic coefficients (see for example [D-S], Sect. XIII.7, p. 1486-1498), but, as it will be demonstrated in this work, (1) possesses many properties which are not present in the general case, thus deserves special attention.

It is well known that (1) is a self-adjoint problem with no boundary conditions at $\pm \infty$ (see for example [C-L], Chap. 7, Exer. 11, or [D-S] Sect. XIII.6, Th. 35). It follows that the spectrum of (1), which from now on we will denote by $S(a, \rho)$, is a subset of the real numbers. Due to the periodicity (see [D-S], Sect. XIII.7, pp. 1486-1498) $S(a, \rho)$ is a union of disjoint nondegenerate closed intervals, the bands. It is easy to see that inf $S(a, \rho)=0$. The set $\mathbf{R}_{+} \backslash S(a, \rho)$ is a (possibly empty) countable union of disjoint open intervals, called gaps. For the "unperturbed" case $a(x) \equiv \rho(x) \equiv 1$, we have $S_{0}=S(1,1)=[0, \infty)$ i.e. there are no gaps (another way to say this is: all gaps are closed), but in general gaps do exist. For example, if $a(x) \rho(x) \equiv 1$, then (1) can be written in the form

$$
L^{2} u=\lambda u, \quad \text { where } \quad L u=-a u^{\prime \prime} .
$$

Now $L$ is a Hill type operator, thus the spectrum of $L$, and therefore the spectrum of $L^{2}$, has gaps and the same is true if we consider small perturbations of this case.

The Green's function $G(x, y ; \lambda)$ associated to (1) satisfies

$$
\left[a(x) G_{x x}(x, y ; \lambda)\right]_{x x}=\lambda \rho(x) G(x, y ; \lambda)+\delta(x-y) .
$$

Notice that we have the symmetries

$$
G(x, y ; \lambda)=G(y, x ; \lambda) \text { and } G(x+b, y+b ; \lambda)=G(x, y ; \lambda) .
$$

For any fixed $\lambda$ the "shift" transformation $(T u)(x)=u(x+b)$ maps solutions of (1) to solutions. Thus $T=T(\lambda)$ is a well-defined linear transformation on the fourth-dimensional vector space of the solutions of (1). As a basis of this space we take the solutions $u_{j}(x ; \lambda), j=1,2,3,4$, such that (primes refer to derivatives with respect to $x$ )

$$
u_{j}^{(k-1)}(0 ; \lambda)=\delta_{j k}, \quad k \neq 4, \quad\left[a u_{j}^{\prime \prime}\right]^{\prime}(0 ; \lambda)=\delta_{j 4} .
$$

If $a(0) \neq 1$, in the case $j=k=3$ it is more appropriate to take $a(0) u_{3}^{\prime \prime}(0 ; \lambda)=1$. The set $\left\{u_{j}\right\}_{j=1}^{4}$ is called a fundamental set of solutions of $(1)$. In the sequel, by a 
slight abuse of terminology, we will sometimes refer to $u_{j}$ as the $j^{\text {th }}$ fundamental solution. A standard Picard iteration argument implies that each $u_{j}(x ; \lambda)$ is entire in $\lambda$ of order $1 / 4$. We identify $T$ with its matrix with respect to the above basis, called Floquet or monodromy matrix, namely (if $a(0) \neq 1$, the third row must be multiplied by $a(0))$

$$
T=\left[t_{j k}\right]=\left[\begin{array}{cccc}
u_{1}(b) & u_{2}(b) & u_{3}(b) & u_{4}(b) \\
u_{1}^{\prime}(b) & u_{2}^{\prime}(b) & u_{3}^{\prime}(b) & u_{4}^{\prime}(b) \\
u_{1}^{\prime \prime}(b) & u_{2}^{\prime \prime}(b) & u_{3}^{\prime \prime}(b) & u_{4}^{\prime \prime}(b) \\
{\left[a u_{1}^{\prime \prime}\right]^{\prime}(b)} & {\left[a u_{2}^{\prime \prime}\right]^{\prime}(b)} & {\left[a u_{3}^{\prime \prime}\right]^{\prime}(b)} & {\left[a u_{4}^{\prime \prime}\right]^{\prime}(b)}
\end{array}\right]
$$

where the dependence in $\lambda$ is suppressed for typographical convenience. Using the properties of the Wronskian of the $u_{j}$ 's we get that

$$
\operatorname{det} T=1 \text {. }
$$

Let $r_{1}, r_{2}, r_{3}, r_{4}$, be the eigenvalues of $T$, sometimes called Floquet multipliers (of course $\left.r_{1} r_{2} r_{3} r_{4}=1\right)$. They are analytic functions of $\lambda$ and the only type of singularities they can have are algebraic ones, i.e. branch points. Except for a discrete set of $\lambda$ 's (we will discuss this set more later), $T$ is similar to a diagonal matrix and its eigenvectors correspond to the Floquet solutions, namely to the solutions $f_{j}, j=1,2,3,4$, of (1) such that

$$
f_{j}(x)=e^{w_{j} x} p_{j}(x), \quad \text { where } \quad r_{j}=e^{w_{j} b}, \quad \text { and } \quad p_{j}(x+b)=p_{j}(x) .
$$

The $w_{j}$ 's are called Floquet exponents or quasimomenta (or even complex rotation numbers, following R. Johnson and J. Moser-see (J-M]). It follows that the spectrum $S(a, \rho)$ is characterized as

$$
S(a, \rho)=\left\{\lambda:\left|r_{j}(\lambda)\right|=1, \quad \text { for some } j\right\},
$$

whereas the Green's function has the form

$$
G(x, y ; \lambda)=\sum_{j, k} c_{j k} f_{j}(x) f_{k}(y), \quad x \leqq y,
$$

where each $f_{j}(x)$ is a Floquet solution belonging to $L^{2}(-\infty, 0)$ and each $f_{k}(y)$ belongs to $L^{2}(0, \infty)$ (see [D-S], Sect. XIII.7.64, Th. 64). By setting $x=y$ in (4) and using (a) the fact that $G(x, x, \lambda)$ is periodic in $x$, and (b) the form of $f_{j}$ given by (3), we obtain an important property of the Floquet multipliers, namely that they occur in pairs of inverses,

$$
r_{1} r_{4}=r_{2} r_{3}=1 \text {. }
$$

It follows from (5) that the characteristic equation of $T$ is an inverse equation, i.e. it has the form

$$
r^{4}-A(\lambda) r^{3}+B(\lambda) r^{2}-A(\lambda) r+1=0,
$$

where $A(\lambda)$ and $B(\lambda)$ are certain invariants of $T$, entire in $\lambda$.

An interesting question is how one can extract $w_{j}(\lambda)$ from the Green's function $G(x, y ; \lambda)$ as Johnson and Moser did for the second order problem. This will show how to define rotation numbers in the cases where $a$ and $\rho$ are almost periodic or ergodic. Such cases are also common in applications. 
It is instructive to see what all these quantities become when $a(x) \equiv \rho(x) \equiv 1$. When we consider a quantity of this (unperturbed) case we index it by 0 . The $u_{j}$ 's become

$$
\begin{array}{ll}
u_{01}(x ; \lambda)=(\cosh s x+\cos s x) / 2, & u_{02}(x ; \lambda)=(\sinh s x+\sin s x) / 2 s, \\
u_{03}(x ; \lambda)=(\cosh s x-\cos s x) / 2 s^{2}, & u_{04}(x ; \lambda)=(\sinh s x-\sin s x) / 2 s^{3}
\end{array}
$$

where $s=\lambda^{1 / 4}$ (notice that $u_{0 j}=u_{04}^{(4-j)}$ ). It follows that

$$
\begin{aligned}
& A_{0}(\lambda)=2\left[\cosh \left(\lambda^{1 / 4} b\right)+\cos \left(\lambda^{1 / 4} b\right)\right], \\
& B_{0}(\lambda)=2+4 \cosh \left(\lambda^{1 / 4} b\right) \cos \left(\lambda^{1 / 4} b\right)
\end{aligned}
$$

and

$$
G_{0}(x, y ; \lambda)=\frac{1}{4 \lambda^{3 / 4}}\left[i e^{i \lambda^{1 / 4}|x-y|}-e^{-\lambda^{1 / 4}|x-y|}\right], \quad 0<\arg \lambda^{1 / 4}<\pi / 2 .
$$

The Riemann surface of the solution $r(\lambda)$ of (6), has four sheets and its branches are $r_{j}(\lambda), j=1,2,3,4$. The only possible $\lambda$ 's for which our equation has not four linearly independent Floquet solutions are the $\lambda$ 's for which the characteristic equation (6) of $T$ has multiple roots. The value $\lambda=0$ is such a $\lambda$. In this case the fundamental solutions can be written down explicitly. It follows that $A(0)=4, B(0)=6$, and that there is only one Floquet solution (namely $u_{1}$ ) while $r(\lambda)$ has always a fourth-root branch point at $\lambda=0$.

If $\lambda \neq 0$, then the characteristic equation of $T$ can only have simple or double roots (otherwise, by $(5) r_{j}(\lambda)=1$, for all $j$, which is impossible because, as we will see below, when $\lambda \neq 0$ is in the spectrum, there is a $j$ for which $\left.r_{j}(\lambda)>1\right)$. Now let $\lambda \neq 0$ be such that (6) has a double root, say $r_{j}$. Then there is one Floquet solution $f_{j}(x+b)=r_{j} f_{j}(x)$ and a solution $g_{j}(x)\left(f_{j}\right.$ and $g_{j}$ are linearly independent $)$ such that $g_{j}(x+b)=r_{j} g_{j}(x)+c_{j} f_{j}(x)$, where the constant $c_{j}$ may be 0 (in this case we say that we have coexistence, i.e. two linearly independent Floquet solutions corresponding to the same multiplier). If $c_{j} \neq 0$, we can say that, for this particular $\lambda, T$ has a Jordan anomaly (this terminology is due to Professor Barry Simon) and that $g_{j}(x)$ is a generalized Floquet solution of $(1)$.

\section{Some Basic Properties of the Fundamental Solutions}

We start with a property of all nontrivial solutions of (1). Its proof is easy and thus omitted.

Proposition 1. Let $u \neq 0$ be a solution of (here a and $\rho$ need not be periodic)

$$
\left(a u^{\prime \prime}\right)^{\prime \prime}=\lambda \rho u, \quad \lambda \neq 0 .
$$

If $-\infty<c<d<\infty$, then each of the functions $u, u^{\prime}, u^{\prime \prime}$ and $\left(a u^{\prime \prime}\right)^{\prime}$ has finitely many zeros in $[c, d]$.

Remark. It is easy to construct an example for which $u(x)=x^{2}+1$ is a solution, thus it can happen that $u^{\prime \prime \prime} \equiv 0$.

The next property of the $u_{j}$ 's plays a crucial role in the spectral theory of (1). The eigenvalue parameter $\lambda$ is assumed positive. This covers all the values in the spectrum $S(a, \rho)$, with trivial exception of 0 . 
Proposition 2. Let $\lambda>0$. If $u_{j}$ is the $j^{\text {th }}$ fundamental solution of (1), then

$$
\lim _{x \rightarrow+\infty} u_{j}(x)=+\infty, \quad \lim _{x \rightarrow-\infty} u_{j}(x)=(-1)^{j-1} \infty .
$$

Furthermore, $u_{2}$ and $u_{4}$ are always increasing, whereas $u_{1}$ and $u_{3}$ are decreasing on $(-\infty, 0)$ and increasing on $(0, \infty)$. trivial.

The proof of this proposition is based on the following lemma whose proof is

Lemma. Let $f \in C^{n}[0, \infty), f^{(k)}(0) \geqq 0,0 \leqq k<n$ and $f^{(n)}(0)>0$, where $n \geqq 1$ is a fixed integer. If $x_{0}>0$ is such that $f\left(x_{0}\right)=0$, then there are numbers $x_{k}, k=1,2, \ldots, n$ such that

$$
0<x_{n}<\cdots<x_{1}<x_{0} \text { and } f^{(k)}\left(x_{k}\right)=0 .
$$

Proof of Proposition 2. Let $f(x)=a(x) u_{1}^{\prime \prime}(x)$. Then

$$
f(0)=f^{\prime}(0)=0, \quad f^{\prime \prime}(x)=\lambda \rho(x) u_{1}(x),
$$

in particular $f^{\prime \prime}(0)=\lambda \rho(0) u_{1}(0)=\lambda \rho(0)>0$. If $x_{2} \in(0, \infty)$ is the smallest zero of $f^{\prime \prime}$, then $x_{2}$ is the smallest positive zero of $u_{1}$ (if $u_{1}$ has positive zeros, then $x_{2}$ is well defined by Proposition 1). Applying the lemma to $u_{1}$, we have that there is a $x_{0} \in\left(0, x_{2}\right)$, such that $u_{1}^{\prime \prime}\left(x_{0}\right)=0$. Thus $f\left(x_{0}\right)=0$ and this contradicts the lemma. Thus $f>0$ on $(0, \infty)$, therefore $u_{1}^{\prime}$ increases on $(0, \infty)$, thus $u_{1}$ increases to $\infty$. The case $x \in(-\infty, 0)$ is reduced to the case $x \in(0, \infty)$ by replacing $x$ by $-x$ in (1).

We mention without proof another property that recalls the oscillation properties of the Sturm-Liouville equations.

Proposition 3. Assume $\lambda<0$. Let $x_{1}<x_{2}<x_{3}<\cdots$, be the positive zeros of $u_{1}(x)$ and $0=x_{1}^{(m)}<x_{2}^{(m)}<x_{3}^{(m)}<\cdots, m=1,2,3$, be the (positive) zeros of $u_{1}^{\prime}(x), u_{1}^{\prime \prime}(x)$ and $\left[a u_{1}^{\prime \prime}\right]^{\prime}(x)$ respectively. We then have that $u_{1}(x)$ has infinitely many positive (and therefore negative, by replacing $x$ by $-x$ ) zeros and the interlacing property holds namely

$$
0<x_{1}<x_{2}^{(3)}<x_{2}^{(2)}<x_{2}^{(1)}<x_{2}<x_{3}^{(3)}<x_{3}^{(2)}<x_{3}^{(1)}<x_{3}<x_{4}^{(3)}<\cdots
$$

Furthermore, all these zeros are simple.

\section{The Band-Gap Structure of the Spectrum}

We start by observing that Proposition 2 of the previous section implies that, if $\lambda>0$, we cannot have $\left|r_{j}(\lambda)\right|=1$, for all $j=1,2,3,4$. Hence, by (5)

$$
\left|r_{j}(\lambda)\right|>1, \quad \text { for some } j \text {, say } j=4 \text {. }
$$

Now, let us assume that $r_{4}(\lambda)$ is not a positive real. Consider the corresponding Floquet solution

$$
f_{4}(x)=e^{w_{4} x} p_{4}(x), \quad w_{4}=\beta_{4}+i \alpha_{4} .
$$


Since $r_{4}=e^{w_{4} b}$, we must have ( $n$ denotes an integer)

$$
\alpha_{4}=\mathfrak{F}\left\{w_{4}\right\} \neq 2 n \pi / b ; \text { in particular } \alpha_{4} \neq 0 .
$$

Since $\lambda$ is real, it follows that the complex conjugate $\overline{f_{4}}$ of $f_{4}$ is also a Floquet solution of (1) and

$$
\mathfrak{R}\left\{f_{4}\right\}=e^{\beta_{4} x}\left[\Re\left\{p_{4}(x)\right\} \cos \left(\alpha_{4} x\right)-\mathfrak{F}\left\{p_{4}(x)\right\} \sin \left(\alpha_{4} x\right)\right]
$$

is a solution too.

Next, observe that $\mathfrak{R}\left\{f_{4}\right\}$ determines the leading behavior of at least two of the fundamental solutions. But, because of (7), $\mathfrak{R}\left\{f_{4}\right\}$ oscillates (alternates sign) no matter how big $x$ is. This is in contradiction with Proposition 2 . Therefore

$$
r_{4}(\lambda)>1, \text { for all } \lambda>0 .
$$

This inequality, together with Proposition 2, imply that, if $\lambda>0$, then $f_{4}(x)$ never vanishes. Changing $x$ to $-x$, we obtain that the same is true about $f_{1}(x)$.

Let us now suppose that $\lambda$ is moving continuously on the positive real axis towards $+\infty$, initially being at 0 , namely at the lowest point of $S(a, \rho)$. If $\lambda$ is sufficiently close to 0 , it must be in $S(a, \rho)$, since there are no isolated points in this spectrum. It follows that, at the beginning of $\lambda$ 's "journey" $r_{2}(\lambda)$ and $r_{3}(\lambda)$ move on the unit circle, always being complex conjugates to each other, while $r_{4}(\lambda)$ is real and always increasing (being, by definition, the largest Floquet multiplier). Initially, i.e. at $\lambda=0$, we have $r_{1}(0)=r_{2}(0)=r_{3}(0)=r_{4}(0)=1$. Now, as long as $r_{2}(\lambda)$ and $r_{3}(\lambda)$ move on the unit circle, they cannot switch direction (see [D-S], Sect. XIII.7, p. 1493). The only time that $\lambda$ will stop being in $S(a, \rho)$ is when $r_{2}(\lambda)$ and $r_{3}(\lambda)$ leave the unit circle. It follows that this can first happen when $r_{2}(\lambda)=r_{3}(\lambda)=-1$. We denote $\lambda_{1}^{\prime}$ the first value of $\lambda$ at which $r_{2}=r_{3}=-1$. Then, as $\lambda$ becomes bigger than $\lambda_{1}^{\prime}, r_{2}(\lambda)$ and $r_{3}(\lambda)$ can "escape" (from the unit circle) into the negative real axis. As $\lambda$ keeps increasing, $r_{2}(\lambda)$ and $r_{3}(\lambda)$ will enter again the unit circle, and the only way to do that is through -1 . We denote $\lambda_{2}^{\prime}$ the corresponding value of $\lambda$ at which $r_{2}$ and $r_{3}$ enter the circle again, with the convention that, if $r_{2}(\lambda)$ and $r_{3}(\lambda)$ stay on the unit circle for all $\lambda \in\left(\lambda_{1}^{\prime}, \lambda_{1}^{\prime}+\varepsilon\right)$, then we set $\lambda_{2}^{\prime}=\lambda_{1}^{\prime}$. The next time they can escape is when they become equal to 1 . We denote $\lambda_{1}$ the corresponding value of $\lambda$. Then $r_{2}(\lambda)$ and $r_{3}(\lambda)$ can escape in the positive real axis. Eventually, as $\lambda$ keeps increasing, they will enter the unit circle again, this time through 1 , and let $\lambda_{2}$ be the corresponding value of $\lambda$, again with the convention that, if $r_{2}$ and $r_{3}$ do not leave the circle, then $\lambda_{2}=\lambda_{1}$.

As $\lambda$ keeps moving to $+\infty$, this process repeats itself again and again. Thus we can define $\lambda_{2 n-1}^{\prime}$ and $\lambda_{2 n}^{\prime}, n=1,2,3, \ldots$ to be the values of $\lambda$ at which $r_{2}(\lambda)$ and $r_{3}(\lambda)$ leave and re-enter (respectively) the unit circle through -1 , for the $n^{\text {th }}$ time. Hence, $r_{2}\left(\lambda_{2 n-1}^{\prime}\right)=r_{3}\left(\lambda_{2 n}^{\prime}\right)=-1$. Of course, $\lambda_{2 n-1}^{\prime} \leqq \lambda_{2 n}^{\prime}$ and our convention is that equality holds if $r_{2}$ and $r_{3}$ do not leave the circle through -1 during their $n^{\text {th }}$ passage. In a similar way we define the numbers $\lambda_{2 n-1}$ and $\lambda_{2 n}, n=1,2,3, \ldots$, with the only difference that we now have $r_{2}\left(\lambda_{2 n-1}\right)=r_{3}\left(\lambda_{2 n}\right)=1$. Since $r_{2}(0)=$ $r_{3}(0)=1$, while $r_{2}(\lambda) \neq 1$ when $\lambda \notin[0, \infty)$, it makes sense to set $\lambda_{0}=0$. Observe that, since the periodic and antiperiodic spectra of (1), which we denote by $S_{p}(a, \rho)$ and $S_{a}(a, \rho)$ respectively, are real and positive, we must have the set equations

$$
S_{p}(a, \rho)=\left\{\lambda_{n}: n=0,1,2,3, \ldots\right\}, \quad \text { and } \quad S_{a}(a, \rho)=\left\{\lambda_{n}^{\prime}: n=1,2,3, \ldots\right\} .
$$

We have thus established the following theorem. 
Theorem 1. The spectrum $S(a, \rho)$ of (1) has the form

$$
S(a, \rho)=\left[0, \lambda_{1}^{\prime}\right] \cup\left[\lambda_{2}^{\prime}, \lambda_{1}\right] \cup\left[\lambda_{2}, \lambda_{3}^{\prime}\right] \cup\left[\lambda_{4}^{\prime}, \lambda_{3}\right] \cup\left[\lambda_{4}, \lambda_{5}^{\prime}\right] \cup \cdots,
$$

where the numbers $\lambda_{n}^{\prime}$ and $\lambda_{n}, n=1,2,3, \ldots$, are defined in the previous paragraph.

If $\lambda \in S(a, \rho)$, then $\left|r_{2}(\lambda)\right|=\left|r_{3}(\lambda)\right|=1$. If $\lambda_{2 n-1}<\lambda<\lambda_{2 n}$, then $0<r_{1}(\lambda)<$ $r_{2}(\lambda)<1<r_{3}(\lambda)<r_{4}(\lambda)$, whereas if $\lambda_{2 n-1}^{\prime}<\lambda<\lambda_{2 n}^{\prime}$, then $r_{3}(\lambda)<-1<r_{2}(\lambda)$ $<0$ and $\left|r_{3}(\lambda)\right| \leqq r_{4}(\lambda)$. (Reminder: $r_{1}(\lambda) r_{4}(\lambda)=r_{2}(\lambda) r_{3}(\lambda)=1$, for all $\left.\lambda \in \mathbf{C}\right)$.

Remarks. (a) At the endpoints $\lambda_{2 n-1}, \lambda_{2 n}$ or $\lambda_{2 n-1}^{\prime}, \lambda_{2 n}^{\prime}(n \geqq 1)$ of a gap, $r_{2}(\lambda)$ and $r_{3}(\lambda)$ have square-root type branch points if and only if the gap is open (otherwise, they do not have any singularity). Notice that $r_{1}(\lambda)$ and $r_{4}(\lambda)$ do not have singularities at the endpoints of the gaps. We remind the reader that, at $\lambda=\lambda_{0}=0$, all $r_{j}$ have a fourth-root branch point.

(b) From Theorem 1 it follows that the bands do not overlap.

Equation (6) implies (by setting $r=1$ ) that the periodic eigenvalues, are the zeros of the entire function

$$
f^{+}(\lambda)=B(\lambda)-2 A(\lambda)+2,
$$

while the antiperiodic eigenvalues are the zeros of (set $r=-1$ in (6))

$$
f^{-}(\lambda)=B(\lambda)+2 A(\lambda)+2 .
$$

The quantity

$$
\Delta(\lambda)=2\left[r_{2}(\lambda)+r_{3}(\lambda)\right]=A(\lambda)-\sqrt{E(\lambda)},
$$

where

$$
E(\lambda)=A(\lambda)^{2}-4 B(\lambda)+8 \quad\left(\text { notice that } E(0)=0, E^{\prime}(0)>0\right)
$$

can be called the discriminant. If $\lambda \geqq 0$, then Theorem 1 implies that $\Delta(\lambda)$ is real (therefore $E(\lambda) \geqq 0$ ) and

$$
|\Delta(\lambda)| \leqq 4 \text { if and only if } \lambda \in S(a, \rho) .
$$

When $\Delta(\lambda)=4, \lambda$ is a periodic eigenvalue and when $\Delta(\lambda)=-4, \lambda$ is an antiperiodic eigenvalue. Notice that $\Delta(\lambda)$ has square-root branch points at the zeros of $E(\lambda)$ of odd multiplicity. Contrary to the case of the Hill's equation, here the periodic (or antiperiodic) eigenvalues alone do not determine $\Delta(\lambda)$.

The entire function $E(\lambda)$ is a little mysterious, since it does not have a counterpart in the second order (i.e. Hill's equation) case. Its relation to the Floquet multipliers is given by the formula

$$
E(\lambda)=\frac{1}{4}\left\{\left[r_{1}(\lambda)+r_{4}(\lambda)\right]-\left[r_{2}(\lambda)+r_{3}(\lambda)\right]\right\}^{2} .
$$

If $a(x) \equiv \rho(x) \equiv 1$, then

$$
f_{0}^{ \pm}(\lambda)=4\left[1 \mp \cosh \left(\lambda^{1 / 4} b\right)\right]\left[1 \mp \cos \left(\lambda^{1 / 4} b\right)\right],
$$


with zeros (of $f_{0}^{+}(\lambda)$ and $f_{0}^{-}(\lambda)$ respectively)

$$
\lambda_{00}=0, \lambda_{0,2 n-1}=\lambda_{0,2 n}=(2 n \pi / b)^{4}, \quad \lambda_{0,2 n-1}^{\prime}=\lambda_{0,2 n}^{\prime}=[(2 n+1) \pi / b]^{4}, n \geqq 1 .
$$

Also,

$$
E_{0}(\lambda)=4\left[\cosh \left(\lambda^{1 / 4} b\right)-\cos \left(\lambda^{1 / 4} b\right)\right]^{2},
$$

with zeros

$$
\tilde{\lambda}_{00}=0, \tilde{\lambda}_{0,2 n-1}, \tilde{\lambda}_{0,2 n}=-4(n \pi / b)^{4}, \quad n \geqq 1
$$

(all zeros are double, except for $\lambda_{00}, \tilde{\lambda}_{00}$ which are simple) and finally

$$
\Delta_{0}(\lambda)=4 \cos \left(\lambda^{1 / 4} b\right) .
$$

Proposition 4. The multiplicity of any zero of $f^{+}(\lambda)$ can be only 1 or 2 . A zero $\lambda^{*}$ of $f^{+}(\lambda)$ is double if and only if $\lambda^{*}=\lambda_{2 n-1}=\lambda_{2 n}$, for some $n \geqq 1$ (i.e. the corresponding gap is closed). In particular 0 is always a simple zero of $f^{+}(\lambda)$. Similar statements are true for $f^{-}(\lambda)$, with the obvious exception that $f^{-}(0) \neq 0$.

Proof. We set $a(x)=1+\tilde{a}(x), \rho(x)=1+\tilde{\rho}(x)$, and introduce $a_{t}(x)=1+t \tilde{a}(x)$ and $\rho_{t}(x)=1+t \tilde{\rho}(x), 0 \leqq t \leqq 1$ (thus $a_{1}=a$ and $\rho_{1}=\rho$ ). Consider also $f_{t}^{+}(\lambda)$, i.e. the entire function whose zeros are the periodic eigenvalues of the problem with coefficients $a_{t}(x)$ and $\rho_{t}(x)$. Now $f_{t}^{+}(\lambda)$ depends continuously in $t$. Therefore the zeros of $f_{t}^{+}(\lambda)$ depend continuously on $t$. On the other hand, (8) implies that the proposition is true for $t=0$. The rest follows by Theorem 1 and a continuity argument (since no band of the spectrum can ever collapse to a single point).

Corollary 1. If $\lambda_{2 n-1} \neq \lambda_{2 n}, n \geqq 1$, then, as $\lambda \rightarrow \lambda_{2 n-1}$,

$$
r_{2}(\lambda)=1-a_{2 n-1} \sqrt{\lambda-\lambda_{2 n-1}}+O\left(\lambda-\lambda_{2 n-1}\right)
$$

and

$$
r_{3}(\lambda)=1+a_{2 n-1} \sqrt{\lambda-\lambda_{2 n-1}}+O\left(\lambda-\lambda_{2 n-1}\right),
$$

where $a_{2 n-1}>0$ and $\sqrt{ }$ denotes the principal branch of the square-root function. The behavior of $r_{2}$ and $r_{3}$ at $\lambda_{2 n}$ is similar. If $\lambda_{2 n-1}=\lambda_{2 n}$, then, as $\lambda \rightarrow \lambda_{2 n-1}$,

$$
r_{2}(\lambda)=1+i b_{2 n-1}\left(\lambda-\lambda_{2 n-1}\right)+O\left[\left(\lambda-\lambda_{2 n-1}\right)^{2}\right]
$$

and

$$
r_{3}(\lambda)=1-i b_{2 n-1}\left(\lambda-\lambda_{2 n-1}\right)+O\left[\left(\lambda-\lambda_{2 n-1}\right)^{2}\right],
$$

where $b_{2 n-1}>0$. The same result (with the necessary modifications) is true for the antiperiodic eigenvalues.

Finally (case $n=0$ ), as $\lambda \rightarrow 0$,

$$
r_{2}(\lambda)=1+i a_{0} \lambda^{1 / 4}+O(\lambda), \quad r_{3}(\lambda)=1-i a_{0} \lambda^{1 / 4}+O(\lambda),
$$

where $a_{0}>0$ and $\lambda^{1 / 4}$ denotes the principal branch of the fourth-root function.

Conjecture 1. For a given periodic (antiperiodic) eigenvalue $\lambda^{*}$, we have coexistence of two linearly independent periodic (antiperiodic) solutions if and only if $\lambda^{*}=$ $\lambda_{2 n-1}=\lambda_{2 n}\left(\lambda^{*}=\lambda_{2 n-1}^{\prime}=\lambda_{2 n}^{\prime}\right)$. This conjecture is in the spirit of the recent work of F. Gesztesy and R. Weikard on Floquet theory (see [G-W]). 
Let us denote the zeros of $E(\lambda)$ by $\tilde{\lambda}_{n}, n=0,1,2,3, \ldots$ counting multiplicities (since it has order $1 / 4$, it must have infinitely many zeros). We always have that $\tilde{\lambda}_{0}=0$ is a simple zero. If $\tilde{\lambda}_{n} \neq 0$ has odd multiplicity, then it is a square-root type branch point where the sheets of $r_{2}$ and $r_{3}$ meet the sheets of $r_{1}$ and $r_{4}$. The following proposition is quite surprising.

Proposition 5. If $\tilde{\lambda}_{n} \neq 0$ is a zero of $E(\lambda)$ of odd multiplicity, then $\tilde{\lambda}_{n}$ is real and negative. Furthermore, if there are functions $a$ and $\rho$ such that their corresponding $E(\lambda)$ has a nonreal zero (necessarily of even multiplicity), then there are different $a$ and $\rho$ such that their corresponding $E(\lambda)$ has a negative zero of multiplicity $2 m, m \geqq 2$.

Proof. Let $\lambda$ be in the open upper half-plane and assume that all $\left|r_{J}(\lambda)\right|=e^{\beta_{J} b}$ are distinct, say $\beta_{1}(\lambda)<\beta_{2}(\lambda)<\beta_{3}(\lambda)<\beta_{4}(\lambda)$.

We now introduce the "shifted" equation

$$
\left(a_{\xi} u^{\prime \prime}\right)^{\prime \prime}=\lambda \rho_{\xi} u, \quad \text { where } \quad a_{\xi}(x)=a(x+\xi), \rho_{\xi}(x)=\rho(x+\xi) .
$$

The Floquet solutions of the shifted equation are

$$
f_{j \xi}(x ; \lambda)=e^{w_{j}(\lambda) x} p_{j}(x+\xi ; \lambda) .
$$

Next, consider the solution $\phi(x ; \lambda ; \xi)$ of the shifted equation that satisfies the conditions

$$
\phi(0 ; \lambda ; \xi)=1, \quad \phi(x ; \lambda ; \xi)=O\left(e^{\beta_{1} x}\right), \quad \text { as } x \rightarrow \infty .
$$

Then, $\phi$ must have the form

$$
\phi(x ; \lambda ; \xi)=\frac{e^{w_{1}(\lambda) x} p_{1}(x+\xi ; \lambda)}{p_{1}(\xi ; \lambda)} .
$$

Differentiating with respect to $x$ we get (primes denote the derivative with respect to the first entry of the function)

$$
\phi^{\prime}(x ; \lambda ; \xi)=\frac{w_{1}(\lambda) e^{w_{1}(\lambda) x} p_{1}(x+\xi ; \lambda)+e^{w_{1}(\lambda) x} p_{1}^{\prime}(x+\xi ; \lambda)}{p_{1}(\xi ; \lambda)}
$$

Finally, for $x=0$ the above formula becomes

$$
\phi^{\prime}(0 ; \lambda ; \xi)=w_{1}(\lambda)+\frac{p_{1}^{\prime}(\xi ; \lambda)}{p_{1}(\xi ; \lambda)} .
$$

The quantity $m(\lambda ; \xi)=\phi^{\prime}(0 ; \lambda ; \xi)$ is an example of a Weyl-Kodaira $m$-function. It is known (see [Y2, J or N, Part II, Sect. 21.4]) that, as a function of $\lambda, m$ is meromorphic in the (open) upper half-plane. On the other hand, the previous equation gives

$$
\frac{1}{b} \int_{0}^{b} m(\lambda ; \xi) d \xi=w_{1}(\lambda)+\frac{1}{b} \int_{0}^{b} \frac{p_{1}^{\prime}(\xi ; \lambda)}{p_{1}(\xi ; \lambda)} d \xi=w_{1}(\lambda) .
$$

It follows that $w_{1}(\lambda)$ is meromorphic and therefore $r_{1}(\lambda)$ is analytic in the (open) upper half-plane, since the only singularities that $r_{1}$ can have are branch points. Then the same is true for $r_{4}(\lambda)=r_{1}(\lambda)^{-1}$ and $r_{2}(\lambda), r_{3}(\lambda)$, because $r_{2}, r_{3}$ have common branch points with $r_{1}$. 
Now, let $\tilde{\lambda}_{n}$ be a zero of $E(\lambda)$ with odd multiplicity. Then it is a branch point of $r_{j}(\lambda)$. It follows that $\tilde{\lambda}_{n}$ must be real. Furthermore $\tilde{\lambda}_{n}$ cannot be positive, since $r_{3}(\lambda)$ and $r_{4}(\lambda)$ do not cross each other as long as $\lambda>0$. Finally, if $\tilde{\lambda}_{n}$ is a nonreal zero of even multiplicity, then by a continuity argument and the fact that the zeros of $E_{0}(\lambda)$ are all real, it follows that there are functions $a$ and $\rho$ for which their corresponding $E(\lambda)$ has a negative zero of even multiplicity $\geqq 4$.

The above proof is inspired by $[\mathrm{J}]$. An immediate consequence of the above proposition is that $r_{j}\left(\tilde{\lambda}_{n}\right)$ is real for all $j$. In the case $a \rho \equiv 1$, it is not hard to see that all the nonzero zeros of $E(\lambda)$ are double.

Conjecture 2. The multiplicities of the zeros of $E(\lambda)$ can be only one or two and, furthermore, (counting multiplicities) they can be arranged so that

$$
0=\tilde{\lambda}_{0}<\tilde{\lambda}_{1} \leqq \tilde{\lambda}_{2}<\tilde{\lambda}_{3} \leqq \tilde{\lambda}_{4}<\tilde{\lambda}_{5} \leqq \tilde{\lambda}_{6}<\cdots
$$

We have coexistence of four (two and two) linearly independent (proper) Floquet solutions at $\lambda=\tilde{\lambda}_{n}$ if and only if $\tilde{\lambda}_{n}$ is a double zero. The intervals $\left(\tilde{\lambda}_{2 n-1}, \tilde{\lambda}_{2 n}\right)$ can be called "pseudogaps" or $\psi$-gaps and (as in the case of the standard gaps) if $\lambda$ is in such an interval, then $r_{j}(\lambda)$ is real, for all $j$ (at a double zero of $E(\lambda)$ the corresponding $\psi$-gap is "closed," i.e. empty). The asymptotics of $r_{j}(\lambda)$, as $\lambda \rightarrow \tilde{\lambda}_{n}$, are similar to the ones given in Corollary 1 for the case $\lambda \rightarrow \lambda_{n}$.

In fact, as $\lambda$ moves on the negative real axis from 0 to $-\infty, r_{1}(\lambda)$ (and $r_{2}$; if $r_{1} \notin \mathbf{R}$, then $r_{2}=\overline{r_{1}}$ ) moves on a spiral (instead of the unit circle which was the case when $\lambda>0$ ) around 0 and inside the unit circle. When $\lambda$ enters a $\psi$-gap, $r_{1}(\lambda)$ leaves the spiral and enters the real axis, and the other $r_{j}$ 's behave similarly.

Indisputable numerical evidence indicates that, generically, the $\psi$-gaps are open.

\section{Some Special Inverse Results}

We start with the asymptotic behavior of the solutions of (1) for large $|\lambda|$, where $\lambda$ can be complex. Assuming that $a, \rho \in C^{2}(\mathbf{R})$, one can write certain solutions of (1) in the form

$$
u=e^{S}
$$

where the asymptotics of $S$ and its derivatives up to order 4 , as $|\lambda| \rightarrow \infty$, are (see [N], Part I, Sect. 4.6)

$$
S^{(m)}(x)=\varepsilon^{-1} S_{0}^{(m)}(x)+S_{1}^{(m)}(x)+\varepsilon S_{2}^{(m)}(x)+o(\varepsilon), \quad \varepsilon^{4}=\frac{1}{\lambda}, \quad m=0,1,2,3,4 .
$$

Substituting this in (1) and comparing powers of $\varepsilon$ we obtain

$$
a\left(S_{0}^{\prime}\right)^{4}=\rho, \quad 2\left(S_{0}^{\prime}\right)^{3} a^{\prime}+6\left(S_{0}^{\prime}\right)^{2} S_{0}^{\prime \prime} a+4\left(S_{0}^{\prime}\right)^{3} S_{1}^{\prime} a=0, \text { etc }
$$

Thus

$$
S_{0}^{\prime}=\left(\frac{\rho}{a}\right)^{1 / 4}, S_{1}=\ln \left(\frac{1}{a^{1 / 8} \rho^{3 / 8}}\right)+C,
$$


and also

$$
S_{2}^{\prime}=\frac{10 a a^{\prime} \rho \rho^{\prime}-13\left(a^{\prime}\right)^{2} \rho^{2}-45 a^{2}\left(\rho^{\prime}\right)+24 a a^{\prime \prime} \rho^{2}+40 a^{2} \rho \rho^{\prime \prime}}{128 a^{7 / 4} \rho^{9 / 4}} .
$$

Therefore

$$
u=\frac{C}{a^{1 / 8} \rho^{3 / 8}} \exp \left[\frac{1}{\varepsilon} \int_{0}^{x}\left(\frac{\rho}{a}\right)^{1 / 4}+\varepsilon S_{2}\right]\left[1+O\left(\varepsilon^{2}\right)\right]
$$

( $\varepsilon$ has four values, thus we get four linearly independent solutions). The results that follow are consequences of this nice formula.

Theorem 2. (a) If $r_{j}(\lambda)$ is a Floquet multiplier of (1), then, as $|\lambda| \rightarrow \infty$,

$$
r_{j}(\lambda)=e^{b i^{1 / 4}+K i^{-1 / 4}}\left[1+O\left(\lambda^{-1 / 2}\right)\right]
$$

where (see (9))

$$
K=S_{2}(b)-S_{2}(0)=\int_{0}^{b} \frac{5\left(a^{\prime}\right)^{2} \rho^{2}+6 a a^{\prime} \rho \rho^{\prime}+5 a^{2}\left(\rho^{\prime}\right)^{2}}{128 a^{7 / 4} \rho^{9 / 4}} d x
$$

(the formula for $r_{j}$ covers all cases, since $\lambda^{1 / 4}$ has four values).

(b) The asymptotics of the periodic eigenvalues are

$$
\lambda_{2 n-1}^{1 / 4}, \lambda_{2 n}^{1 / 4}=\frac{2 n \pi}{b}+\frac{K}{2 n \pi}+O\left(n^{-2}\right) .
$$

(c) The asymptotics of the antiperiodic eigenvalues are

$$
\left(\lambda_{2 n-1}^{\prime}\right)^{1 / 4},\left(\lambda_{2 n}^{\prime}\right)^{1 / 4}=\frac{(2 n+1) \pi}{b}+\frac{K}{(2 n+1) \pi}+O\left(n^{-2}\right) .
$$

(d) If $\tilde{\lambda}_{n}$ is the $n^{\text {th }}$ zero of $E(\lambda)$ and we set $\tilde{\lambda}_{n}=-s_{n}^{4}$, then

$$
s_{2 n-1}, s_{2 n}=\frac{\sqrt{2} n \pi}{b}+\frac{K}{\sqrt{2} n \pi}+O\left(n^{-2}\right) .
$$

Proof. Part (a) follows from (10). The second formula for $K$ follows from (9) and integration by parts. Then parts (b), (c) and (d) follow from (a). For example, (d) follows from the fact that $\tilde{\lambda}_{n}$ is a solution of the equation $r_{1}(\lambda)=r_{2}(\lambda)$.

Remark. Notice that

$$
K \geqq 0, \quad K=0 \quad \text { if and only if } \quad a^{\prime} \equiv \rho^{\prime} \equiv 0
$$

and, due to our normalization, $a^{\prime} \equiv \rho^{\prime} \equiv 0$ implies $a \equiv \rho \equiv 1$.

The following corollary follows immediately from Theorem 2 and the above remark. It recalls an old theorem of Borg for the Hill equation.

Corollary 2. (a) If the equation (1) has infinitely many periodic (antiperiodic) eigenvalues in common with the unperturbed equation then $a \equiv \rho \equiv 1$.

(b) Let $E(\lambda)$ correspond to (1). If $E(\lambda)$ and $E_{0}(\lambda)$ have infinitely many zeros in common, then again $a \equiv \rho \equiv 1$. 
We finish with a theorem which also recalls another old result of Borg concerning the Hill's operator, namely that if all the spectral gaps are closed, then the coefficient function in the Hill's equation is constant.

Theorem 3. If $S(a, \rho)=[0, \infty)$ and the nonzero zeros of $E(\lambda)$ have even multiplicities, then $a(x) \equiv \rho(x) \equiv 1$.

Proof. First observe that 0 is the only branch point of the Floquet multipliers, therefore

$$
r_{j}(\lambda)=g\left(\lambda^{1 / 4}\right)
$$

where $g(z)$ is entire. Since $r_{j}(\lambda)$ has order $1 / 4, g(z)$ is of order 1 . Now $r_{j}(\lambda)$ has no zeros $\left(r_{1} r_{2} r_{3} r_{4}=1\right)$, and this implies that $g(z)=c_{1} e^{c_{2} z}\left(c_{1}, c_{2}\right.$ are constants $)$, i.e.

$$
r_{j}(\lambda)=c_{1} e^{c_{2} \lambda^{1 / 4}}
$$

Thus, part (a) of Theorem 2 implies that $K=0$ (and $c_{1}=c_{2}=1$ ), hence $a^{\prime} \equiv$ $\rho^{\prime} \equiv 0$.

Theorem 3 can give us some hints of how to formulate the general (periodic) inverse problem for (1).

\section{Remarks on the General Inverse Problem}

A natural question arising from this work is how to formulate the inverse problem for (1).

Suppose we consider the equation $(1)$ on $L^{2}(-\infty, \xi) \oplus L^{2}(\xi, \infty)$ with boundary condition $u(\xi)=u^{\prime \prime}(\xi)=0$. Then the essential spectrum of this new problem is again $S(a, \rho)$, but this new problem may also have point spectrum, say $\left\{\mu_{n}(\xi)\right\}_{n}$. Each $\mu_{n}(\xi)$ must be real by self-adjointness. Furthermore, all $\mu_{n}(\xi)$ must be strictly positive, and it is not hard to see that the only places where these $\mu_{n}$ 's can appear are the spectral gaps.

Using the fundamental solutions we can construct a function $F(\lambda ; \xi)$, analytic in $\lambda$, such that $\lambda=\mu_{n}$ is a zero of $F(\lambda)$, for all $n$. In fact, if for convenience we set $\xi=0$, we can take

$$
F(\lambda)=\left[H\left(r_{1}\right) K\left(r_{2}\right)-H\left(r_{2}\right) K\left(r_{1}\right)\right]\left[H\left(r_{3}\right) K\left(r_{4}\right)-H\left(r_{4}\right) K\left(r_{3}\right)\right],
$$

where

$$
H\left(r_{j}\right)=\left|\begin{array}{ccc}
t_{22}-r_{j} & t_{21} & t_{24} \\
t_{32} & t_{31} & t_{34} \\
t_{42} & t_{41} & t_{44}-r_{j}
\end{array}\right|, \quad K\left(r_{j}\right)=\left|\begin{array}{ccc}
t_{22}-r_{j} & t_{23} & t_{24} \\
t_{32} & t_{33}-r_{j} & t_{34} \\
t_{42} & t_{43} & t_{44}-r_{j}
\end{array}\right|,
$$

and $t_{j k}$ is given by (2).

The "conjugate" function $F^{*}(\lambda)$ of $F(\lambda)$ is

$$
F^{*}(\lambda)=\left[H\left(r_{1}\right) K\left(r_{3}\right)-H\left(r_{3}\right) K\left(r_{1}\right)\right]\left[H\left(r_{2}\right) K\left(r_{4}\right)-H\left(r_{4}\right) K\left(r_{2}\right)\right],
$$

so that $F(\lambda) F^{*}(\lambda)$ is entire. But there are zeros other than the $\mu_{n}$ 's. In fact every zero of $E(\lambda)$ is a zero of $F(\lambda) F^{*}(\lambda)$. Furthermore, if $\lambda^{*}$ is a double periodic or antiperiodic eigenvalue, then $H\left(r_{2}\right)=K\left(r_{2}\right)=0$, thus $\lambda^{*}$ is a zero of $F(\lambda) F^{*}(\lambda)$. 
In the case $a(x) \rho(x) \equiv 1$ we have seen that (1) can be written as

$$
L^{2} u=\lambda u, \quad \text { where } \quad L u=-a u^{\prime \prime} .
$$

We set

$$
z=\sqrt{\lambda}, \quad \text { where } \quad \mathfrak{R}\{\sqrt{\lambda}\} \geqq 0
$$

and consider the problems

$$
L v=z v,
$$

with solutions $v_{1}, v_{2}$ such that $v_{1}(0)=v_{2}^{\prime}(0)=1, v_{1}^{\prime}(0)=v_{2}(0)=0$, and

$$
L w=-z w,
$$

with solutions $w_{1}, w_{2}$ such that $w_{1}(0)=w_{2}^{\prime}(0)=1, w_{1}^{\prime}(0)=w_{2}(0)=0$ (in fact, for $\lambda \neq 0, v_{1}, v_{2}, w_{1}$ and $w_{2}$ are linearly independent solutions of (1)). It follows that

$$
F(\lambda)=C \sqrt{\lambda}\left[w_{1}(b)+w_{2}^{\prime}(b)-v_{1}(b)-v_{2}^{\prime}(b)\right]^{4} v_{2}(b) w_{2}(b) v_{1}^{\prime}(b) w_{1}^{\prime}(b)
$$

where $C$ is a constant. Of course, $F^{*}(\lambda)=-F(\lambda)$. Finally, notice that

$$
\left[w_{1}(b)+w_{2}^{\prime}(b)-v_{1}(b)-v_{2}^{\prime}(b)\right]^{4}=c E(\lambda)^{2} .
$$

If, in particular, $a(x) \equiv \rho(x) \equiv 1$, then

$$
F_{0}(\lambda)=-F_{0}^{*}(\lambda)=\frac{\lambda}{16} E_{0}(\lambda)^{2} \sqrt{f_{0}^{+}(\lambda) f_{0}^{-}(\lambda)} .
$$

By applying a continuity argument to the above formulas it might be possible to show that, in general, $F(\lambda)$ has two zeros in each gap, one of which is a $\mu_{n}$.

Next we need to derive determinant formulas (i.e. multiplicative analogs of trace formulas - see for example [C] or [G-H-S-Z]). Here is a strategy for deriving such a formula.

Since $F^{*}(\lambda ; \xi) F(\lambda ; \xi)$ is entire of order $1 / 4$ we can write

$$
F^{*}(\lambda ; \xi) F(\lambda ; \xi)=-C \lambda^{7} \prod_{0 \neq j \in Z}\left[1-\frac{\lambda}{\tau_{j}(\xi)}\right],
$$

where the functional $C=C[a, \rho]$ is invariant under shifts (i.e. $C\left[a_{\xi}, \rho_{\xi}\right]=C[a, \rho]$ ) and the set $\left\{\tau_{J}(\xi)\right\}$ contains the set $\left\{\mu_{n}(\xi)\right\}$. Likewise (11) implies

$$
F_{0}^{*}(\lambda) F_{0}(\lambda)=-C_{0} \lambda^{7} \prod_{0 \neq j \in Z}\left[1-\frac{\lambda}{\tau_{0_{j}}}\right],
$$

where, for $j>0, \tau_{0 j}$ is the $j^{\text {th }}$ nonzero zero of $f_{0}^{+}(\lambda) f_{0}^{-}(\lambda)$ counting multiplicities, while, for $j<0, \tau_{0 j}$ is the $j^{\text {th }}$ nonzero zero of $E_{0}(\lambda)^{4}$ counting multiplicities. These zeros are given explicitly in Sect. 3. Dividing the above two equations and letting $|\lambda| \rightarrow \infty$ we obtain

$$
\lim _{|\lambda| \rightarrow \infty} \frac{F_{0}^{*}(\lambda) F_{0}(\lambda)}{F^{*}(\lambda ; \xi) F(\lambda ; \xi)}=\frac{C_{0}}{C} \prod_{0 \neq j \in Z} \frac{\tau_{j}(\xi)}{\tau_{0 j}} .
$$


On the other hand, using the asymptotics of $u_{j}(b ; \lambda)$ and $r_{j}(\lambda)$ for large $|\lambda|$ (see Theorem 2 and the discussion in the beginning of Sect. 4) we get

$$
\lim _{|\lambda| \rightarrow \infty} \frac{F_{0}^{*}(\lambda) F_{0}(\lambda)}{F^{*}(\lambda ; \xi) F(\lambda ; \xi)}=\frac{1}{[a(\xi) \rho(\xi)]^{2}} .
$$

Combining the above two equations we have

$$
\prod_{0 \neq j \in Z} \frac{\tau_{j}(\xi)}{\tau_{0 j}}=\frac{C}{C_{0}} \frac{1}{[a(\xi) \rho(\xi)]^{2}} .
$$

This is a determinant formula. Notice that the product is independent of $\xi$ if and only if $a(x) \rho(x) \equiv 1$. This agrees with the fact that in the Sturm-Liouville case, the regularized product of the Dirichlet and Neumann eigenvalues is independent of shifts.

One can do a little more. First

$$
E(\lambda)^{4} f^{+}(\lambda) f^{-}(\lambda)=D_{1} \lambda^{5} \prod_{0 \neq j \in Z}\left[1-\frac{\lambda}{\sigma_{j}}\right],
$$

where $D_{1}=D_{1}[a, \rho]$ is again invariant under shifts and, for $j>0, \sigma_{j}$ is the $j^{\text {th }}$ nonzero zero of $f^{+}(\lambda) f^{-}(\lambda)$ counting multiplicities, while, for $j<0, \sigma_{j}$ is the $j^{\text {th }}$ nonzero zero of $E(\lambda)^{4}$ counting multiplicities. In particular

$$
E_{0}(\lambda)^{4} f_{0}^{+}(\lambda) f_{0}^{-}(\lambda)=256 C_{0} \lambda^{5} \prod_{0 \neq j \in Z}\left[1-\frac{\lambda}{\tau_{0_{j}}}\right]
$$

Thus

$$
\lim _{|\lambda| \rightarrow \infty} \frac{E_{0}(\lambda)^{4} f_{0}^{+}(\lambda) f_{0}^{-}(\lambda)}{E(\lambda)^{4} f^{+}(\lambda) f^{-}(\lambda)}=\frac{256 C_{0}}{D_{1}} \prod_{0 \neq j \in Z} \frac{\sigma_{j}}{\tau_{0 j}} .
$$

Now, the asymptotics of $u_{j}(b ; \lambda)$ and $r_{j}(\lambda)$ for large $|\lambda|$ give

$$
\lim _{|\lambda| \rightarrow \infty} \frac{E_{0}(\lambda)^{4} f_{0}^{+}(\lambda) f_{0}^{-}(\lambda)}{E(\lambda)^{4} f^{+}(\lambda) f^{-}(\lambda)}=D_{2}
$$

and combining the above two equations

$$
\prod_{0 \neq j \in Z} \frac{\sigma_{j}}{\tau_{0 j}}=\frac{D_{1} D_{2}}{256 C_{0}} .
$$

Finally, combining the above with (12) we get a second determinant formula, namely

$$
\prod_{0 \neq j \in Z} \frac{\tau_{j}(\xi)}{\sigma_{j}}=\frac{256 C}{D_{1} D_{2}} \frac{1}{[a(\xi) \rho(\xi)]^{2}},
$$

where $256 C / D_{1} D_{2}$ is independent of $\xi$.

Acknowledgements. The author wants to express his gratitude (i) to Professor Alan Elcrat of the Wichita State University for bringing his attention to the literature related to the Euler-Bernoulli equation, and (ii) to Professor Elias Koukoutsis of the National Technical University of Athens 
for helping him to run Mathematica, a valuable tool in the derivation of some formulas contained in the present work.

\section{Bibliography}

[B] Barcilon, V.: Sufficient Conditions for the Solution of the Inverse Problem for a Vibrating Beam. Inverse Problems 3, 181-193 (1987)

[C-L] Coddington, E.A., Levinson, N.: Theory of Ordinary Differential Equations. Malabar, Florida: Robert E. Krieger Publishing Company, 1987

[C] Craig, W.: The Trace Formula for Schrödinger Operators on the Line. Commun. Math. Phys. 126, 379-407 (1989)

[D-S] Dunford, N., Schwartz, I.T.: Linear Operators. Part II: Spectral Theory; Self Adjoint Operators in Hilbert Space. New York: Wiley Classics Library Edition, 1988

[G-H-S-Z] Gesztesy, F., Holden, H., Simon, B., Zhao, Z.: Trace Formulae and Inverse Spectral Theory for Schrödinger Operators, Bull. Am. Math. Soc. (New Series) 29, 250-255 (1993)

[G-W] Gesztesy, F., Weikard, R.: Floquet Theory Revisited. Preprint, 1994

[G] Gladwell, G.M.L.: Inverse Problems in Vibration. Boston, Martinus Nijhoff Publishers, 1986

[J] Johnson, R.: m-Functions and Floquet Exponents for Linear Differential Systems. Annali di Mathematica pura ed applicata (IV), Vol. CXLVII, 211-248 (1987)

[J-M] Johnson, R., Moser, J.: The Rotation Number for Almost Periodic Potentials. Commun. Math. Phys. 84, 403-438 (1982): Erratum: Commun. Math. Phys. 90, 317-318 (1983)

[Mc] McLaughlin, J.R.: On Constructing Solutions to an Inverse Euler-Bernoulli Problem. In: F. Santosa, et al. (editors), Inverse Problems of Acoustic and Elastic Waves, Philadelphia: SIAM, pp. 341-347 (1984)

[M] Miller, R.E.: The Eigenvalue Problem for a Class of Long, Thin Elastic Structures with Periodic Geometry. Quarterly of Applied Mathematics, Vol. LII, No. 2, 261-282, (June 1994)

[N] Naimark, M.A.: Linear Differential Operators, Part I \& II. New York: Frederick Ungar Publishing Co., 1967 \& 1968

[T-Y] Timoshenko, S., Young, D.H.: Elements of Strength of Materials. 5th ed., Princeton, NJ: D. Van Nostrand Company, Inc., 1968

[Y1] Yurko, V.A.: A Problem in Elasticity Theory, translated from Prikl. Matem. Mekhan. 54, No. 6, 998-1002 (1990) PMM U.S.S.R. 54, No. 6, 820-824 (1990)

[Y2] Yurko, V.A.: Recovery of Differential Operators from the Weyl Matrix. Translated from Dokl. Akad. Nauk U.S.S.R. 313, No. 6, (1990), Sov. Math. Dokl. 42, No. 1, 229-233 (1991)

Communicated by B. Simon 
